\title{
The Association Analysis of GPNMB rs156429 With Clinical Manifestations in Chinese Population With Parkinson's Disease
}

\author{
Jin Liu ${ }^{1 \dagger}$, Gen $\mathrm{Li}^{1 \dagger}$, Yixi He ${ }^{1}$, Guiying He ${ }^{1}$, Pingchen Zhang ${ }^{1}$, Xin Shen ${ }^{1}$, Weishan Zhang ${ }^{1}$, \\ Shengdi Chen ${ }^{1 *}$, Shishuang Cui ${ }^{2 *}$ and Yuyan Tan ${ }^{1 *}$ \\ ${ }^{1}$ Department of Neurology \& Collaborative Innovation Center for Brain Science, Ruijin Hospital Affiliated to Shanghai Jiao \\ Tong University School of Medicine, Shanghai, China, ${ }^{2}$ Department of Geriatrics, Ruijin Hospital Affiliated to Shanghai Jiao \\ Tong University School of Medicine, Shanghai, China
}

OPEN ACCESS

Edited by:

Yidong Bai,

The University of Texas Health Science Center at San Antonio, United States

Reviewed by:

Andrew DeWan,

Yale University, United States

Yuhu Zhang,

Guangdong Provincial People's

Hospital, China

*Correspondence:

Shengdi Chen

chensd@rih.com.cn

Shishuang Cui

cuis2007@163.com

Yuyan Tan

yuyantan00@126.com

${ }^{\dagger}$ These authors have contributed equally to this work

Specialty section:

This article was submitted to

Genetics of Aging,

a section of the journal

Frontiers in Genetics

Received: 05 March 2020

Accepted: 29 July 2020

Published: 02 September 2020

Citation:

Liu J, Li G, He Y, He G, Zhang P, Shen $X$, Zhang $W$, Chen $S$, Cui $S$ and Tan Y (2020) The Association Analysis

of GPNMB rs 156429 With Clinical Manifestations in Chinese Population With Parkinson's Disease.

Front. Genet. 11:952.

doi: 10.3389/fgene.2020.00952
Background: The mechanisms of Parkinson's disease (PD) include complicated genetic factors. The roles of newly found risk genes need to be further verified among different ethnicities. In a two-stage meta-analysis, single nucleotide polymorphism (SNP) of rs156429 in glycoprotein non-metastatic melanoma protein B (GPNMB) was reported to be associated with PD. So far clinical studies have focused on association between rs156429 and PD onset, however there is little evidence linking rs156429 with PD symptoms.

Objective: This study aimed to investigate the possible association of GPNMB rs156429 with PD manifestations among southeastern Chinese people.

Methods: Demographic variables, disease-related factors, and motor and non-motor assessments of 511 PD patients were collected. Polymerase chain reaction (PCR) and SNaPshot technique were used to detect GPNMB rs156429. The associations of rs156429 with PD rating scales and clinical manifestations were analyzed by Kruskal-Wallis test and logistic regression model separately.

Results: Kruskal-Wallis test and logistic regression model failed to reveal an association between GPNMB rs156429 and scores from Montreal Cognitive Assessment (MoCA) $(p=0.037 ; p=1.000$ after correction), and pain symptoms of 511 PD patients ( $p$ $=0.008, \mathrm{OR}=0.59,95 \% \mathrm{Cl}=0.40-0.87$, overdominant model after adjustment; $p=0.168$ after correction, overdominant model after adjustment). However, further analysis based on genders showed that GPNMB rs156429 might have a trend for being associated with cognitive dysfunction (Mini-Mental State Examination (MMSE), $p=0.064$ after correction; MoCA, $p=0.064$ after correction) and pain symptoms $(p=0.063$ after correction, overdominant model after adjustment) in female PD patients but not male patients.

Conclusions: This study revealed that GPNMB rs156429 might have a trend for being associated with cognitive dysfunction and pain symptoms of female PD patients in the southeastern Chinese population. Further studies from a larger sample size are needed to confirm these findings.

Keywords: Parkinson's disease, single nucleotide polymorphisms, GPNMB, genetics, clinical manifestation 


\section{INTRODUCTION}

Parkinson's disease (PD) is one of the most common neurodegenerative disorders (Saeed et al., 2017). It is predominantly characterized by motor symptoms including bradykinesia, resting tremor, rigidity, and posture instability. In addition, non-motor manifestations like rapid eye movement sleep behavior disorder (RBD), hyposmia, depression, and autonomic dysfunction, are also prevalent in $\mathrm{PD}$ patients (Saeed et al., 2017). The mechanisms of PD are complex. Besides aging and neurotoxins, genetic factors are quite crucial for disease onset (Pang et al., 2019).

By now, several classic PD-associated loci like SNCA, GBA, LRRK2, PARK7, and PINK1 have been broadly verified by both basic and clinical studies (Pang et al., 2017). With the application of genome-wide association studies (GWAS), many new loci have been identified (Do et al., 2011; Nalls et al., 2011, 2014). A two-stage meta-analysis found that glycoprotein nonmetastatic melanoma protein B $(G P N M B) / 7$ p15 was associated with PD onset (International Parkinson's Disease Genomics Consortium (IPDGC) Wellcome Trust Case Control Consortium 2 (WTCCC2)., 2011). Several subsequent studies indicated the relationship between single nucleotide polymorphism (SNP) of GPNMB rs156429 and PD, in both the Scandinavian population (Pihlstrom et al., 2013) and the central Chinese population (Liu et al., 2015). In Liu's report, there were significant differences between male Chinese PD participants and healthy controls in both genotype $(p=0.01)$ and allele distribution $(p=0.01$, $\mathrm{OR}=0.67)$ of $\mathrm{rs} 156429$, indicating that GPNMB rs156429 could have a protective role in male Chinese patients with $\mathrm{PD}$ (Liu et al., 2015). However, results were inconsistent in other European-descended populations (Hernandez et al., 2012; SotoOrtolaza et al., 2013; Kara et al., 2014) and the southwestern Chinese population ( $\mathrm{Xu}$ et al., 2016), which failed to find the association of GPNMB rs156429 with PD. More related clinical studies with larger sample sizes are urgently needed. In despite of contradictory clinical results, researchers found that GPNMB was

\footnotetext{
Abbreviations: AD, Alzheimer's disease; ADL, activity of daily living; ALS, Amyotrophic lateral sclerosis; AMPA, $\alpha$-amino-3-hydroxy-5-methylisoxazole-4propionate; BPI, Brief Pain Inventory; CI, Confidence interval; COMT, catecholO-methyltransferase; CSF, cerebrospinal fluid; EOPD, early-onset Parkinson's disease; ESS, Epworth Sleeping Scale; FAAH, fatty acid amide hydrolase; FSS, Fatigue Severity Scale; GPNMB, glycoprotein non-metastatic melanoma protein B; GWAS, genome-wide association studies; HAMA, Hamilton Anxiety Rating Scale; HAMD, Hamilton Depression Rating Scale; HWE, HardyWeinberg equilibrium; H-Y staging, Hoehn-Yahr staging; IBD, Inflammatory bowel disease; LOPD, late-onset Parkinson's disease; LPS, lipopolysaccharide; MDS, Movement Disorder Society; MDS-UPDRS, the Movement Disorder Society Unified Parkinson's Disease Rating Scale; MMSE, Mini-Mental State Examination; MoCA, Montreal Cognitive Assessment; MPTP, 1-methyl-4-phenyl1,2,3,6-tetrahydropyridine; NMSS, Non-Motor Symptoms Scale; OR, Odds ratio; PCR, polymerase chain reaction; PD, Parkinson's disease; PDQ-39, 39-item Parkinson's disease Questionnaire; PDSS, Parkinson's disease Sleep Scale; PSG, polysomnography; RBD, Rapid Eye Movement Sleep Behavior Disorder; RBDHK, Rapid Eye Movement Sleep Behavior Disorder Questionnaire-Hong Kong Version; SCN9A, sodium voltage-gated channel alpha subunit 9; SCOPA-AUT, Scales for Outcomes in Parkinson's disease-Autonomic Questionnaire; SD, standard deviation; SE, standard error; SNP, single nucleotide polymorphism; SS-16, Sniffin' Sticks-16.
}

both transcriptionally and expressively elevated in the substantia nigra areas of PD patients (Murthy et al., 2017; Moloney et al., 2018; Neal et al., 2018). GPNMB could involve in regulation of inflammation and immune systems (Budge et al., 2018), which also participated in PD pathogenesis. In addition, one study found that overexpression of GPNMB could protect against neurodegeneration changes induced by neurotoxins (Budge et al., 2020), indicating that GPNMB could serve as an emerging target for neurodegenerative diseases (Budge et al., 2018).

As for association between GPNMB rs156429 and PD symptoms, there were few related findings. The results of one study conducted among elderly American people with mild Parkinsonian signs showed that this SNP polymorphism was associated with the number of steps taken in a timed $2.4-\mathrm{m}$ gait trial (Shulman et al., 2014). Another study showed that rs156429 had no association with anxiety and depression symptoms in southwestern Chinese PD patients (Xu et al., 2016). To date, there has been no study focusing on the relationship between this SNP and detailed motor and non-motor symptoms of PD. Therefore, in this study, we aimed to investigate the association of GPNMB rs156429 with clinical manifestations of PD in the southeastern Chinese population, providing clinical evidences for exploring potential molecular mechanisms of GPNMB rs156429 function in PD.

\section{METHODS}

\section{Study Population}

PD was diagnosed by movement disorder physicians according to diagnostic criteria established by movement disorders society (MDS) (Postuma et al., 2015). A total of 511 PD patients were recruited from 2016 to 2018 at Ruijin Hospital. Participants with atypical parkinsonism, secondary parkinsonism, and other movement disorders were excluded from this study. In addition, patients with comorbidities that might affect the reliable completion of clinical assessments were also excluded, such as severe visual or hearing impairment, and inability to write or speak. All participants in the study were fully informed, and signed consent forms. This study was approved by the ethics committee of Ruijin Hospital affiliated to Shanghai Jiao Tong University School of Medicine.

\section{Assessments}

Clinical assessments were completed, and blood samples were collected. Demographic information includes age, gender, education level, and family history. We also analyzed the composition of PD subtypes as late-onset PD (LOPD) or earlyonset PD (EOPD). Based on previous reports, LOPD was defined as the PD patient older than 45 years old at the time of first diagnosis (González-Del Rincón et al., 2013). Clinical assessments include Hoehn-Yahr (H-Y) staging; the Movement Disorder Society Unified Parkinson's Disease Rating Scale (MDSUPDRS) (Martinez-Martin et al., 2015); Non-Motor Symptoms Scale (NMSS) (Martinez-Martin et al., 2009); 39-item Parkinson's disease Questionnaire (PDQ-39) (Galeoto et al., 2018); Sniffin' Sticks-16 (SS-16) (Chen et al., 2015); Hamilton Anxiety Rating Scale (HAMA) (Kummer et al., 2010); Hamilton Depression 
Rating Scale (HAMD) (Kummer et al., 2010); Brief Pain Inventory (BPI) (Lin et al., 2016); Rapid Eye Movement Sleep Behavior Disorder Questionnaire-Hong Kong Version (RBDHK) (Shen et al., 2014); Parkinson's disease Sleep Scale (PDSS) (Selvaraj and Keshavamurthy, 2016); Fatigue Severity Scale (FSS) (Fu et al., 2017); Epworth Sleeping Scale (ESS) (Ataide et al., 2014); Scales for Outcomes in Parkinson's disease-Autonomic Questionnaire (SCOPA-AUT) (Visser et al., 2004); Mini-Mental State Examination (MMSE); and Montreal Cognitive Assessment (MoCA) Beijing Version (Hoops et al., 2009). Disease-related changes in non-motor function, activity of daily living (ADL), and motor function were assessed with MDS-UPDRS parts 1, 2, and 3. General life quality was assessed by PDQ-39. The overall non-motor burden was measured by NMSS. Depression and anxiety levels were assessed by HAMD and HAMA, respectively. Pain symptoms were measured by BPI. Sleep disturbance was assessed by PDSS. Fatigue was measured by FSS, and somnolence was measured by ESS. Autonomic function was assessed by SCOPA-AUT. Cognitive status was assessed by MMSE and MoCA Beijing Version. Olfactory function was assessed by SS16 , and hyposmia was determined when the score of SS-16 was $<8.3$ points (Chen et al., 2015). RBD was assessed by RBD$\mathrm{HK}$, and probable RBD was diagnosed according to previous literature (Shen et al., 2014). Other clinical symptoms, such as dysphagia, sialorrhea, constipation, nocturia, etc., as shown in Supplementary Table 2, were defined as present or absent by two other movement disorder physicians (not those who did the scale assessments).

\section{DNA Preparations and Genotyping}

Peripheral blood samples were collected, and DNA was extracted from leukocytes by using phenol-chloroform isopropyl alcohol method. Primers were designed by Primer 5 (version 5.00, PREMIER Biosoft International). After purification of polymerase chain reaction (PCR) products by both phosphorylase (FastAP, Applied biosystems) and exonuclease I (EXO I, Applied biosystems), extension reaction was done by SNaPshot Multiplex kit of ABI. The extended products were further purified by phosphorylase (FastAP, Applied biosystems), and sampled by ABI3730xl (Applied biosystems). The results of SNP typing were analyzed by genemap 4.0 (Applied biosystems).

The primer information is as follows:

Forward: TCC TGG GGT GTT TGA ATC ATA AG

Reverse: TGT GTT CAC ACA AAA TGT GGG ATT A

Elongation: TGC TTT TTA GAA AAA TAT CAG GAA C.

\section{Statistical Analysis}

Both R (version 3.5.1) and RStudio (version 1.1.463, RStudio, Inc.) were applied for statistical analysis. The packages used included readxl package (version 1.2.0), CATT package (version 2.0), base package (version 3.5.1), and stats package (version 3.5.1) (package link: https://cran.r-project.org/web/packages/ index.html). Chi-square test or Fisher exact test was adopted to assess Hardy-Weinberg equilibrium (HWE), and compare the differences in gender, PD subtype, education level, Hoehn-Yahr staging, and family history among GPNMB rs156429 subgroups. ANOVA was applied to test the differences of age at interview.
TABLE 1 | Demographic information of GPNMB rs156429 in PD patients.

\begin{tabular}{lcccc}
\hline & $\begin{array}{c}\text { TT genotype } \\
(\boldsymbol{n}=\mathbf{3 0 4})\end{array}$ & $\begin{array}{c}\text { CT genotype } \\
(\boldsymbol{n}=\mathbf{1 7 6})\end{array}$ & $\begin{array}{c}\text { CC genotype } \\
(\boldsymbol{n}=\mathbf{3 1})\end{array}$ & $\boldsymbol{p}$-value \\
& & & & \\
\hline Gender (female, $n, \%)$ & $133(43.75)$ & $80(45.45)$ & $18(58.06)$ & 0.790 \\
Age at interview (mean & $62.24(10.47)$ & $63.92(11.37)$ & $63.86(9.11)$ & 0.115 \\
\pm SD) & & & & \\
Subtype (LOPD, $n, \%)$ & $280(92.11)$ & $165(93.75)$ & $30(96.77)$ & 0.657 \\
Education (n, \%) & & & & 0.638 \\
Illiterate & $11(3.73)$ & $2(1.18)$ & $1(3.45)$ & \\
$\leq 6$ years & $45(15.25)$ & $27(15.88)$ & $6(20.69)$ & \\
$6-12$ years & $158(53.56)$ & $93(54.71)$ & $17(58.62)$ & \\
$>12$ years & $81(27.46)$ & $48(28.24)$ & $5(17.24)$ & \\
Hoehn-Yahr staging & & & & 0.423 \\
$(n, \%)$ & & & & \\
1 & $80(28.99)$ & $40(25.00)$ & $8(27.59)$ & \\
1.5 & $44(15.94)$ & $24(15.00)$ & $5(17.24)$ & \\
2 & $119(43.12)$ & $78(48.75)$ & $10(34.48)$ & \\
2.5 & $12(4.35)$ & $6(3.75)$ & $3(10.34)$ & \\
3 & $14(5.07)$ & $10(6.25)$ & $1(3.45)$ & \\
4 & $6(2.17)$ & $0(0.00)$ & $1(3.45)$ & \\
5 & $1(0.36)$ & $2(1.25)$ & $1(3.45)$ & \\
Family history $(n, \%)$ & $31(10.20)$ & $26(14.94)$ & $4(12.90)$ & 0.303 \\
\hline
\end{tabular}

LOPD, late-onset Parkinson's disease; SD, standard deviation.

For continuous outcomes (such as MMSE, MoCA, BPI, HAMD, etc.), we first checked whether those data followed the normal distribution. If so, ANOVA was applied. Otherwise, KruskalWallis test was applied for non-parametric variables. Some PD symptoms, such as hyposmia, probable RBD, etc., were recorded as categorical variables. To investigate the association between GPNMB rs156429 and those symptoms, logistic regression model was adopted, and four genetic models (additive model, dominant model, recessive model, and overdominant model) were analyzed. These models were all further adjusted by HoehnYahr staging and gender. Odds ratio (OR), 95\% confidence interval (CI), and $p$-value (two-tailed test) were computed. The $p$-values were further assessed by Bonferroni correction of multiple tests (Bonferroni correction of multiple tests was conducted in 32 rating scales and sub-scales, such as SS16, HAMA, HAMD, MDS-UPDRS parts 1, 2, and 3, etc.; and 21 symptoms, such as dysphagia, sialorrhea, constipation, nocturia, etc).

\section{RESULTS}

A total of 511 PD participants were recruited in this study. There were 280 male PD patients (54.79\%) and 231 female patients (45.21\%). Three hundred and four of total recruited PD patients were TT genotype (59.49\%), 176 were CT genotype $(34.44 \%)$ and 31 were CC genotype (6.07\%). GPNMB rs156429 was tested in $\operatorname{HWE}(p=0.851)$. There was no statistical difference in gender, age at interview, PD subtype, education level, HoehnYahr staging, and family history in the three genotypes of PD patients (Table 1). 
The detailed PD rating scale information in all three genotypes of rs156429 was analyzed (Supplementary Table 1). All scores did not follow the normal distribution, thus Kruskal-Wallis test was applied to analyze the association of rating scales scores and GPNMB rs156429 genotypes. There was no significant association between GPNMB rs156429 and PD rating scales ( $p$ $=0.037$, MoCA; $p=1.000$ after correction, MoCA) (Table 2). Multiple pairwise-comparison was further applied to investigate the differences of rating scales among genotypes of GPNMB rs156429. Similarly, MoCA scores failed to pass Bonferroni correction between TT and CC genotypes of PD patients ( $p=0.034$, MoCA; $p=1.000$ after correction, MoCA).

Four genetic models (additive model, dominant model, recessive model, and overdominant model) were applied to investigate the association between $\mathrm{PD}$ symptoms and GPNMB rs156429 polymorphism (Supplementary Table 2). Pain symptoms were shown to have a trend for being associated with GPNMB rs156429 in dominant model ( $p=0.021$, OR = $1.56,95 \% \mathrm{CI}=1.07-2.28$, dominant model after adjustment; $p=0.441$ after correction, dominant model after adjustment) and overdominant model $(p=0.008, \mathrm{OR}=0.59,95 \% \mathrm{CI}=$ $0.40-0.87$, overdominant model after adjustment; $p=0.168$ after correction, overdominant model after adjustment) (Table 3, Supplementary Table 2). The inheritance pattern of GPNMB rs156429 is not clear, and determination of inheritance pattern that a trait will follow is still unknown. Thus, in different genetic models, the genotype might show different or even opposite ORs. Considering the small sample size of monocentric PD participants in our study, the association between pain and GPNMB rs156429 in PD was still highly expected in future studies with more participants.

Previous literature reported that GPNMB rs156429 could have a protective role in male Chinese patients with $\mathrm{PD}$ (Liu et al., 2015). Therefore, we analyzed data in male and female patients separately. Both MMSE and MoCA had a trend in association analysis with GPNMB rs156429 genotypes in female PD patients $(p=0.002$, MMSE; $p=0.064$ after correction, MMSE; $p=0.002$, MoCA; $p=0.064$ after correction, MoCA) (Supplementary Table 3), so did pain symptoms in association analysis with $\mathrm{rs} 156429$ in female PD patients ( $p$ $=0.016, \mathrm{OR}=1.99,95 \% \mathrm{CI}=1.14-3.49$, dominant model after adjustment; $p=0.336$ after correction, dominant model after adjustment; $p=0.003$, OR $=0.41,95 \% \mathrm{CI}=0.22-$ 0.73 , overdominant model after adjustment; $p=0.063$ after correction, overdominant model after adjustment) (Table 3, Supplementary Table 4). For male PD patients, no statistical differences in PD symptoms were found among three rs156429 genotypes (Supplementary Table 5), or in four genetic models (Supplementary Table 6).

\section{DISCUSSION}

In this study, we revealed for the first time that GPNMB rs156429 polymorphism might have a trend for being associated with cognitive dysfunction and pain symptoms among southeastern female Chinese PD patients but not male patients. These findings could provide evidence that GPNMB rs156429 might have different effects on PD symptoms between female and male patients.

$G P N M B$ was identified as one of the newly found risk loci of PD in a two-stage meta-analysis study, and SNP rs156429 was considered to be the most relevant candidate for PD [International Parkinson's Disease Genomics Consortium (IPDGC) and Wellcome Trust Case Control Consortium 2 (WTCCC2), 2011]. There is minimal supporting literature referring to the association of GPNMB rs156429 with PD symptoms. One study revealed that rs 156429 was associated with motor traits in older populations with mild parkinsonian signs (Shulman et al., 2014). Another result suggested that this SNP had no association with anxiety and depression symptoms in PD patients (Xu et al., 2016). As for gender factor, previous literature reported that GPNMB rs156429 might have a protective role in male Chinese PD patients (Liu et al., 2015). In our study, we did not find genotype distribution differences of GPNMB rs156429 between male and female PD patients. However, analysis by genders showed that this SNP might have a trend for being associated with cognitive dysfunction and pain symptoms in female PD populations. These clinical results could serve as clinical evidence for further exploring potential molecular mechanisms of GPNMB rs156429 in PD, especially its roles in PD pathogenesis and symptoms associated with gender differences.

GPNMB, also known as osteoactivin, is a glycoprotein widely expressed in a mass of tissues with multiple physiological functions. In addition to functioning as a metastatic mediator (Zhou et al., 2012; Maric et al., 2013), GPNMB could also play an important role in mediating bone mineral deposition (Abdelmagid et al., 2008; Zhuo and Zhou, 2016), contributing to tissue damage and inflammation (Ahn et al., 2002; Ripoll et al., 2007), and functioning in neuronal survival and neuroprotection (Huang et al., 2012; Srinivasan et al., 2016; Neal et al., 2018). So far, GPNMB has been reported to be involved in cancer diseases (Onaga et al., 2003; Kuan et al., 2006; Rose et al., 2010), non-alcoholic steatohepatitis (Katayama et al., 2015), inflammatory bowel disease (IBD), Niemann Pick disease-type C (Marques et al., 2016), Gaucher disease (Murugesan et al., 2018), amyotrophic lateral sclerosis (ALS) (Tanaka et al., 2012; Nagahara et al., 2015) and PD. In normal rat brain, GPNMB was widely expressed in cerebral cortex, striatum, olfactory bulb, hippocampus and other brain areas (Huang et al., 2012). In PD cases, it was reported that the transcription and expression levels of GPNMB were both increased in substantia nigra (Murthy et al., 2017; Moloney et al., 2018; Neal et al., 2018). Additionally, GPNMB was shown to interact with CD44, and CD44 expression was also highly increased after lipopolysaccharide (LPS) administration in rat substantia nigra (Ailane et al., 2013) and 1-methyl-4phenyl-1,2,3,6-tetrahydropyridine (MPTP) treatment in mice (Kurkowska-Jastrzebska et al., 1999). A recent paper also found that overexpression of GPNMB could protect against neurodegeneration changes induced by MPTP (Budge et al., 2020). These evidences indicated that GPNMB could serve as an emerging target for neurodegenerative diseases (Budge et al., 2018). 
TABLE 2 | The association between PD rating scales and genotype of GPNMB rs156429 in PD patients.

\begin{tabular}{|c|c|c|c|c|c|}
\hline & \multirow{2}{*}{$\begin{array}{c}p \text {-value } \\
\text { TT vs. CT vs. CC } \\
\text { ( } n=304 \text { vs. } 176 \text { vs. } 31 \text { ) }\end{array}$} & \multirow{2}{*}{$\begin{array}{c}p \text {-value }{ }^{a} \\
\text { TT vs. CT vs. CC } \\
\text { ( } n=304 \text { vs. } 176 \text { vs. } 31 \text { ) }\end{array}$} & \multicolumn{3}{|c|}{$p$-value (multiple pairwise-comparison between groups) } \\
\hline & & & $\begin{array}{c}\text { TT vs. CT } \\
(n=304 \text { vs. } 176)\end{array}$ & $\begin{array}{c}\text { TT vs. CC } \\
(n=304 \text { vs. } 31)\end{array}$ & $\begin{array}{c}\text { CT vs. CC } \\
(n=176 \text { vs. } 31)\end{array}$ \\
\hline SS-16 & 0.277 & 1.000 & 0.343 & 0.343 & 0.544 \\
\hline HAMA & 0.625 & 1.000 & 0.725 & 0.595 & 0.595 \\
\hline HAMD & 0.690 & 1.000 & 0.884 & 0.643 & 0.643 \\
\hline $\mathrm{BPI}$ & 0.200 & 1.000 & 0.321 & 0.321 & 0.602 \\
\hline RBD-HK & 0.848 & 1.000 & 0.838 & 0.838 & 0.838 \\
\hline PDSS & 0.643 & 1.000 & 0.925 & 0.925 & 0.962 \\
\hline PDQ39 & 0.441 & 1.000 & 0.618 & 0.815 & 0.815 \\
\hline FSS & 0.738 & 1.000 & 0.910 & 0.910 & 0.949 \\
\hline ESS & 0.538 & 1.000 & 0.968 & 0.473 & 0.473 \\
\hline MDS-UPDRS & 0.649 & 1.000 & 0.641 & 0.641 & 0.641 \\
\hline PARTI & 0.929 & 1.000 & 0.973 & 0.973 & 0.973 \\
\hline PART II & 0.561 & 1.000 & 0.590 & 0.590 & 0.590 \\
\hline PART III & 0.792 & 1.000 & 0.745 & 0.745 & 0.745 \\
\hline NMSS & 0.804 & 1.000 & 0.830 & 0.830 & 0.830 \\
\hline cardiovascular & 0.876 & 1.000 & 0.770 & 0.770 & 0.770 \\
\hline sleep & 0.623 & 1.000 & 0.688 & 0.688 & 0.704 \\
\hline mood disorder & 0.993 & 1.000 & 0.983 & 0.983 & 0.983 \\
\hline delusion & 0.884 & 1.000 & 0.982 & 0.946 & 0.946 \\
\hline attention & 0.785 & 1.000 & 0.965 & 0.775 & 0.775 \\
\hline gastrointestinal & 0.526 & 1.000 & 0.544 & 0.544 & 0.544 \\
\hline urinary & 0.920 & 1.000 & 0.918 & 0.918 & 0.918 \\
\hline sexual dysfunction & 0.737 & 1.000 & 0.908 & 0.908 & 0.908 \\
\hline others & 0.749 & 1.000 & 0.805 & 0.801 & 0.801 \\
\hline SCOPA-AUT & 0.944 & 1.000 & 0.978 & 0.978 & 0.978 \\
\hline gastrointestinal & 0.851 & 1.000 & 0.792 & 0.792 & 0.792 \\
\hline urinary & 0.973 & 1.000 & 0.927 & 0.927 & 0.927 \\
\hline cardiovascular & 0.908 & 1.000 & 0.847 & 0.847 & 0.847 \\
\hline skin & 0.648 & 1.000 & 0.705 & 0.663 & 0.663 \\
\hline sexual dysfunction & 0.942 & 1.000 & 0.898 & 0.898 & 0.898 \\
\hline drug usage & 0.536 & 1.000 & 0.569 & 0.569 & 0.569 \\
\hline MMSE & 0.059 & 1.000 & 0.516 & 0.055 & 0.055 \\
\hline MoCA & 0.037 & 1.000 & 0.392 & 0.034 & 0.062 \\
\hline
\end{tabular}

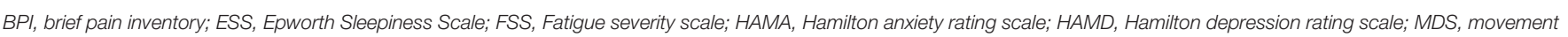

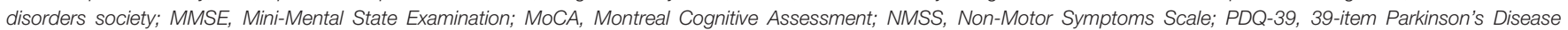

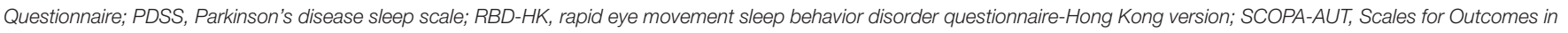
Parkinson's Disease-Autonomic questionnaire; SS-16, Sniffin' Sticks 16; UPDRS, Unified Parkinson's Disease Rating Scale.

${ }^{a} p$-value after Bonferroni correction.

Bold fonts: $p<0.05$.

Nearly $50 \%$ of PD patients were affected by cognitive dysfunction symptoms within 10 years of diagnosis (WilliamsGray et al., 2013). However, both the severity and timing vary among PD patients. Therefore, it will be of great clinical benefits for physicians to early identify patients at risk or at early stages of cognitive dysfunction. The precise mechanisms of cognitive impairment in PD still remain unknown, but it was widely acknowledged that the combination of pathological protein accumulations (alpha synuclein, tau, and amyloid beta) and neurotransmitter changes plays an important role in cognitive involvement (Weil et al., 2018). The association between GPNMB and PD cognitive function has not been reported, but its function in Alzheimer's disease (AD), has been profoundly studied. Firstly, GPNMB was found to be elevated in both cerebrospinal fluid (CSF) and brain samples of sporadic AD patients compared with cognitively healthy controls (Huttenrauch et al., 2018). This study further found that GPNMB was partially involved in microglia activation. Another study revealed that overexpressing GPNMB in transgenic mice could improve some hippocampal memory tests, along with increased levels of $\alpha$-amino-3-hydroxy-5methylisoxazole-4-propionate (AMPA) receptor subunit GluA1 in the hippocampus (Murata et al., 2015). In our study, we discovered that GPNMB rs156429 might have a trend for being associated with cognitive dysfunction in female PD patients. 
TABLE 3 | The association between pain symptoms and genetic models of GPNMB rs156429 in PD patients.

\begin{tabular}{|c|c|c|c|c|c|c|c|c|c|c|c|c|c|c|}
\hline & \multicolumn{7}{|c|}{ Genetic model } & \multicolumn{7}{|c|}{ Genetic model with adjustment } \\
\hline & \multicolumn{7}{|c|}{ Dominant model } & \multicolumn{7}{|c|}{ Dominant model (adjusted) $^{a}$} \\
\hline & Beta & SE & Power & $p$-value & OR & $95 \% \mathrm{Cl}$ & $p$-value ${ }^{c}$ & Beta & SE & Power & $p$-value & OR & $95 \% \mathrm{Cl}$ & $p$-value ${ }^{c}$ \\
\hline PD patients & \multicolumn{7}{|c|}{ Overdominant model } & \multicolumn{7}{|c|}{ Overdominant model (adjusted) ${ }^{a}$} \\
\hline \multirow[t]{3}{*}{$(n=511)$} & Beta & SE & Power & $p$-value & OR & $95 \% \mathrm{Cl}$ & $p$-value ${ }^{c}$ & Beta & SE & Power & $p$-value & OR & $95 \% \mathrm{Cl}$ & $p$-value ${ }^{c}$ \\
\hline & Beta & SE & power & $p$-value & OR & $95 \% \mathrm{Cl}$ & $p$-value ${ }^{c}$ & beta & SE & power & $p$-value & OR & $95 \% \mathrm{Cl}$ & $p$-value ${ }^{c}$ \\
\hline & 0.684 & 0.272 & 0.115 & 0.012 & 1.98 & $(1.17,3.39)$ & 0.252 & 0.687 & 0.285 & 0.133 & 0.016 & 1.99 & $(1.14,3.49)$ & 0.336 \\
\hline Female PD patients & \multicolumn{7}{|c|}{ Overdominant model } & \multicolumn{7}{|c|}{ Overdominant model (adjusted) ${ }^{b}$} \\
\hline$(n=231)$ & Beta & SE & Power & $p$-value & OR & $95 \% \mathrm{Cl}$ & $p$-value ${ }^{c}$ & Beta & SE & Power & $p$-value & OR & $95 \% \mathrm{Cl}$ & $p$-value ${ }^{c}$ \\
\hline & -0.835 & 0.285 & 0.093 & 0.003 & 0.43 & $(0.25,0.75)$ & 0.063 & -0.898 & 0.301 & 0.095 & 0.003 & 0.41 & $(0.22,0.73)$ & 0.063 \\
\hline
\end{tabular}

Cl, confidence interval; OR, odds ratio; SE, standard error.

${ }^{a}$ Hoehn-Yahr staging and gender were taken as adjustments.

${ }^{b}$ Hoehn-Yahr staging was taken as adjustment.

${ }^{c} p$-value after Bonferroni correction.

Bold fonts: $p<0.05$.

Therefore, the exact function of GPNMB in cognitive fields, and determination whether it functions differently between male and female, deserve further detailed investigations.

Pain, as a common non-motor symptom of PD, could greatly affect patients' quality of life. Studies proved that pain thresholds of PD tended to decrease, but this cause was not sufficient enough to explain pain development (Zambito Marsala et al., 2011). The mechanisms of pain symptoms in PD are quite complex, involving changes of cerebral brain, spinal cord, and peripheral nociceptive compartments (Rukavina et al., 2019). Several SNP variations have been identified to be involved in pain perception in PD patients like rs4680 and rs6267 of catechol-O-methyltransferase gene (COMT) (Lin et al., 2017), rs6746030 of sodium voltage-gated channel alpha subunit 9 gene (SCN9A), rs324419 of fatty acid amide hydrolase gene (FAAH) (Greenbaum et al., 2012), and PINK1 (Gierthmuhlen et al., 2009). But GPNMB rs156429 has not yet been reported. Evidence suggests that the loss of dopaminergic neurons could lead to nociceptive hypersensitivity. One previous study reported that GPNMB inhibition could attenuate nociception in neuropathic pain mouse model (Hou et al., 2015). Our results indicated that GPNMB rs156429 might have a trend for being associated with pain symptoms in female PD patients. Therefore, our findings provided clinical evidence that GPNMB rs156429 might be involved in PD pain symptoms, especially in female patients, suggesting a possible biological implication of GPNMB rs156429 in PD manifestation development.

There were quite few literatures exploring possible mechanisms of GPNMB and gender differences. One study suggested that GPNMB could be an androgen-dysregulated gene (Tsui et al., 2012), and another indicated that estrogen was necessary but not sufficient for the expression of GPNMB (Prizant et al., 2016). In Liu's study, it was found that GPNMB rs156429 might have a protective role in male Chinese PD patients referring to PD onset when compared with healthy controls (Liu et al., 2015). They reported that male PD patients had significant lower frequency of $G$ allele. In our study, we focused on exploring the possible effects of GPNMB rs156429 on PD symptoms. Therefore, healthy participants were not included. We applied covariance analysis to analyze the associations between scores of MMSE/MoCA and GPNMB rs156429 alleles in PD patients based on genders. We found that gender, rs156429 genotypes, and the interactions of gender and rs156429 genotypes all had statistically significant effects on MMSE and MoCA $(p<0.001)$. We then used Mantel-Haenszel statistics to investigate the association between pain symptoms and rs156429 alleles in PD patients based on genders. We found that $\mathrm{T}$ allele might be a risk factor for pain symptoms in female PD patients (OR $=1.14,95 \% \mathrm{CI}=1.02-1.29)$. Our study and Liu's report focused on different aspects of GPNMB rs156429 and PD. G allele might be protective to male PD patients referring to $\mathrm{PD}$ onset in Liu's report, while $\mathrm{T}$ allele was likely to be a risk factor for pain symptoms in female PD patients in our study. These were not contradictory. According to these two studies, GPNMB rs156429 might have gender-based association or trend of association with PD onset or some PD symptoms, which could enable us to investigate why and how GPNMB rs156429 functions differently in PD based on genders.

There were some limitations in our study. Firstly, as a single centered study, the sample size of this study $(N=511)$ was not large. In female PD patients, we found that $p$-values after Bonferroni correction failed to have statistical significances ( $p=0.064$ after correction, MMSE; $p=0.064$ after correction, MoCA; pain symptoms, $p=0.063$ after correction, overdominant model after adjustment). Subsequent studies with larger sample size are urgently needed. Secondly, we did not perform more objective tests to assess PD and its clinical symptoms, such as polysomnography (PSG) to diagnose RBD, etc. Thirdly, other loci of GPNMB were not included in this study. Fourthly, other confounding factors, such as course of disease, etc., were 
not taken into account in this study. Those limitations are recommended to be considered in futures studies.

In conclusion, our study for the first time found that GPNMB rs156429 might have a trend for being associated with cognitive dysfunction and pain symptoms in the southeastern female Chinese PD population. Further studies of a larger sample size are needed to confirm these findings.

\section{DATA AVAILABILITY STATEMENT}

The raw data supporting the conclusions of this article will be made available by the authors, without undue reservation.

\section{ETHICS STATEMENT}

The studies involving human participants were reviewed and approved by the ethics committee of Ruijin Hospital affiliated to Shanghai Jiao Tong University School of Medicine. The patients/participants provided their written informed consent to participate in this study.

\section{AUTHOR CONTRIBUTIONS}

$\mathrm{PZ}, \mathrm{XS}$, and WZ collected and sorted the data of PD patients. $\mathrm{YH}$ and GH completed the genetic analysis. GL and JL performed the

\section{REFERENCES}

Abdelmagid, S. M., Barbe, M. F., Rico, M. C., Salihoglu, S., Arango-Hisijara, I., Selim, A. H., et al. (2008). Osteoactivin, an anabolic factor that regulates osteoblast differentiation and function. Exp. Cell Res. 314, 2334-2351. doi: 10.1016/j.yexcr.2008.02.006

Ahn, J. H., Lee, Y., Jeon, C., Lee, S. J., Lee, B. H., Choi, K. D., et al. (2002). Identification of the genes differentially expressed in human dendritic cell subsets by cDNA subtraction and microarray analysis. Blood 100, 1742-1754. doi: 10.1182/blood.V100.5.1742.h81702001742_1742_1754

Ailane, S., Long, P., Jenner, P., and Rose, S. (2013). Expression of integrin and CD44 receptors recognising osteopontin in the normal and LPS-lesioned rat substantia nigra. Eur. J. Neurosci. 38, 2468-2476. doi: 10.1111/ejn.12231

Ataide, M., Franco, C. M., and Lins, O. G. (2014). Daytime sleepiness in Parkinson's disease: perception, influence of drugs, and mood disorder. Sleep Disord. 2014:939713. doi: 10.1155/2014/939713

Budge, K. M., Neal, M. L., Richardson, J. R., and Safadi, F. F. (2018). Glycoprotein NMB: an emerging role in neurodegenerative disease. Mol. Neurobiol. 55, 5167-5176. doi: 10.1007/s12035-017-0707-Z

Budge, K. M., Neal, M. L., Richardson, J. R., and Safadi, F. F. (2020). Transgenic overexpression of GPNMB protects against MPTP-induced neurodegeneration. Mol. Neurobiol. 57, 2920-2933. doi: 10.1007/s12035-020-01921-6

Chen, W., Kang, W. Y., Chen, S., Wang, Y., Xiao, Q., Wang, G., et al. (2015). Hyposmia correlates with SNCA variant and non-motor symptoms in Chinese patients with Parkinson's disease. Parkinsonism Relat. Disord. 21, 610-614. doi: 10.1016/j.parkreldis.2015.03.021

Do, C. B., Tung, J. Y., Dorfman, E., Kiefer, A. K., Drabant, E. M., Francke, U., et al. (2011). Web-based genome-wide association study identifies two novel loci and a substantial genetic component for Parkinson's disease. PLoS Genet. 7:e1002141. doi: 10.1371/journal.pgen.1002141

Fu, R., Cui, S. S., Du, J. J., Huang, P., He, Y. C., Gao, C., et al. (2017). Validation of the Parkinson fatigue scale in Chinese Parkinson's disease patients. Brain Behav. 7:e00712. doi: 10.1002/brb3.712 statistical analysis and drafted the manuscript. SCh, SCu, and YT designed and supervised this study, double-checked the statistical analysis, and revised the manuscript. All authors contributed to the article and approved the submitted version.

\section{FUNDING}

This work was supported by grants from the National Natural Science Foundation of China (grant numbers 81430022, 81771374, 91332107, 81371407, and 81971183), Shanghai Municipal Science and Technology Major Project (2018SHZDZX05), Foundation of Shanghai Municipal Education Commission (2017NKX001), and Shanghai Sailing Program (19YF1429900).

\section{ACKNOWLEDGMENTS}

We sincerely thank all the patients who have participated in this study.

\section{SUPPLEMENTARY MATERIAL}

The Supplementary Material for this article can be found online at: https://www.frontiersin.org/articles/10.3389/fgene. 2020.00952/full\#supplementary-material

Galeoto, G., Colalelli, F., Massai, P., Berardi, A., Tofani, M., Pierantozzi, M., et al. (2018). Quality of life in Parkinson's disease: Italian validation of the Parkinson's disease questionnaire (PDQ-39-IT). Neurol. Sci. 39, 1903-1909. doi: 10.1007/s10072-018-3524-x

Gierthmuhlen, J., Lienau, F., Maag, R., Hagenah, J. M., Deuschl, G., Fritzer, E., et al. (2009). Somatosensory processing in a German family with PINK1 mutations: its potential role in Parkinson disease. J. Neurol. Neurosurg. Psychiatry 80, 571-574. doi: 10.1136/jnnp.2008.158659

González-Del Rincón, M., de L., Monroy Jaramillo, N., Suárez Martínez, A. I., Yescas Gómez, P., Boll Woehrlen, M. C., López López, M., et al. (2013). The L444P GBA mutation is associated with early-onset Parkinson's disease in Mexican mestizos. Clin. Genet. 84, 386-387. doi: 10.1111/cge.12084

Greenbaum, L., Tegeder, I., Barhum, Y., Melamed, E., Roditi, Y., and Djaldetti, R. (2012). Contribution of genetic variants to pain susceptibility in Parkinson disease. Eur. J. Pain 16, 1243-1250. doi: 10.1002/j.1532-2149.2012.00134.x

Hernandez, D. G., Nalls, M. A., Ylikotila, P., Keller, M., Hardy, J. A., Majamaa, K., et al. (2012). Genome wide assessment of young onset Parkinson's disease from Finland. PLOS ONE 7:e41859. doi: 10.1371/journal.pone.00 41859

Hoops, S., Nazem, S., Siderowf, A. D., Duda, J. E., Xie, S. X., Stern, M. B., et al. (2009). Validity of the MoCA and MMSE in the detection of MCI and dementia in Parkinson disease. Neurology 73, 1738-1745. doi: 10.1212/WNL.0b013e3181c34b47

Hou, L., Zhang, Y., Yang, Y., Xiang, K., Tan, Q., and Guo, Q. (2015). Intrathecal siRNA against GPNMB attenuates nociception in a rat model of neuropathic pain. J. Mol. Neurosci. 55, 533-540. doi: 10.1007/s12031-014-0379-3

Huang, J. J., Ma, W. J., and Yokoyama, S. (2012). Expression and immunolocalization of Gpnmb, a glioma-associated glycoprotein, in normal and inflamed central nervous systems of adult rats. Brain Behav. 2, 85-96. doi: 10.1002/brb3.39

Huttenrauch, M., Ogorek, I., Klafki, H., Otto, M., Stadelmann, C., Weggen, S., et al. (2018). Glycoprotein NMB: a novel Alzheimer's disease associated marker expressed in a subset of activated microglia. Acta Neuropathol. Commun. 6:108. doi: 10.1186/s40478-018-0612-3 
International Parkinson's Disease Genomics Consortium (IPDGC) and Wellcome Trust Case Control Consortium 2 (WTCCC2). (2011). A two-stage metaanalysis identifies several new loci for Parkinson's disease. PLoS Genet. 7:e1002142. doi: 10.1371/journal.pgen.1002142

Kara, E., Xiromerisiou, G., Spanaki, C., Bozi, M., Koutsis, G., Panas, M., et al. (2014). Assessment of Parkinson's disease risk loci in Greece. Neurobiol. Aging 35, 442.e9-e16. doi: 10.1016/j.neurobiolaging.2013.07.011

Katayama, A., Nakatsuka, A., Eguchi, J., Murakami, K., Teshigawara, S., Kanzaki, M., et al. (2015). Beneficial impact of Gpnmb and its significance as a biomarker in nonalcoholic steatohepatitis. Sci. Rep. 5:16920. doi: 10.1038/srep16920

Kuan, C. T., Wakiya, K., Dowell, J. M., Herndon, J. E. 2nd, Reardon, D. A., Graner, M. W., et al. (2006). Glycoprotein nonmetastatic melanoma protein, B., a potential molecular therapeutic target in patients with glioblastoma multiforme. Clin. Cancer Res. $12(7 \mathrm{Pt} 1), \quad 1970-1982$. doi: 10.1158/1078-0432.CCR-05-2797

Kummer, A., Cardoso, F., and Teixeira, A. L. (2010). Generalized anxiety disorder and the hamilton anxiety rating scale in Parkinson's disease. Arq. Neuropsiquiatr. 68, 495-501. doi: 10.1590/S0004-282X2010000400005

Kurkowska-Jastrzebska, I., Wronska, A., Kohutnicka, M., Czlonkowski, A., and Czlonkowska, A. (1999). The inflammatory reaction following 1-methyl-4phenyl-1,2,3, 6-tetrahydropyridine intoxication in mouse. Exp. Neurol. 156, 50-61. doi: 10.1006/exnr.1998.6993

Lin, C. H., Chaudhuri, K. R., Fan, J. Y., Ko, C. I., Rizos, A., Chang, C. W., et al. (2017). Depression and catechol-O-methyltransferase (COMT) genetic variants are associated with pain in Parkinson's disease. Sci. Rep. 7:6306. doi: 10.1038/s41598-017-06782-z

Lin, X. J., Yu, N., Lin, X. G., Zhang, Y. F., Chen, Y., Zhang, K., et al. (2016). A clinical survey of pain in Parkinson's disease in Eastern China. Int. Psychogeriatr. 28, 283-289. doi: 10.1017/S1041610215001659

Liu, Z. H., Guo, J. F., Li, K., Wang, Y. Q., Kang, J. F., Wei, Y., et al. (2015). Analysis of several loci from genome-wide association studies in Parkinson's disease in mainland China. Neurosci. Lett. 587, 68-71. doi: 10.1016/j.neulet.2014.12.027

Maric, G., Rose, A. A., Annis, M. G., and Siegel, P. M. (2013). Glycoprotein nonmetastatic $b$ (GPNMB): A metastatic mediator and emerging therapeutic target in cancer. OncoTargets Ther. 6, 839-852. doi: 10.2147/OTT.S44906

Marques, A. R., Gabriel, T. L., Aten, J., van Roomen, C. P., Ottenhoff, R., Claessen, N., et al. (2016). Gpnmb is a potential marker for the visceral pathology in niemann-pick type C disease. PLoS ONE 11:e0147208. doi: 10.1371/journal.pone. 0147208

Martinez-Martin, P., Rodriguez-Blazquez, C., Abe, K., Bhattacharyya, K. B., Bloem, B. R., Carod-Artal, F. J., et al. (2009). International study on the psychometric attributes of the non-motor symptoms scale in Parkinson disease. Neurology 73 , 1584-1591. doi: 10.1212/WNL.0b013e3181c0d416

Martinez-Martin, P., Rodriguez-Blazquez, C., Mario, A., Arakaki, T., Arillo, V. C., Chana, P., et al. (2015). Parkinson's disease severity levels and MDSunified Parkinson's disease rating scale. Parkinsonism Relat. Disord. 21, 50-54. doi: 10.1016/j.parkreldis.2014.10.026

Moloney, E. B., Moskites, A., Ferrari, E. J., Isacson, O., and Hallett, P. J. (2018). The glycoprotein GPNMB is selectively elevated in the substantia nigra of Parkinson's disease patients and increases after lysosomal stress. Neurobiol. Dis. 120, 1-11. doi: 10.1016/j.nbd.2018.08.013

Murata, K., Yoshino, Y., Tsuruma, K., Moriguchi, S., Oyagi, A., Tanaka, H., et al. (2015). The extracellular fragment of GPNMB (glycoprotein nonmelanosoma protein, B., osteoactivin) improves memory and increases hippocampal GluA1 levels in mice. J. Neurochem. 132, 583-594. doi: 10.1111/jnc. 13010

Murthy, M. N., Blauwendraat, C., Guelfi, S., Hardy, J., Lewis, P. A., and Trabzuni, D. (2017). Increased brain expression of GPNMB is associated with genome wide significant risk for Parkinson's disease on chromosome 7p15.3. Neurogenetics 18, 121-133. doi: 10.1007/s10048-017-0514-8

Murugesan, V., Liu, J., Yang, R., Lin, H., Lischuk, A., Pastores, G., et al. (2018). Validating glycoprotein non-metastatic melanoma B (gpNMB, osteoactivin), a new biomarker of Gaucher disease. Blood Cells Mol. Dis. 68, 47-53. doi: 10.1016/j.bcmd.2016.12.002

Nagahara, Y., Shimazawa, M., Tanaka, H., Ono, Y., Noda, Y., Ohuchi, K., et al. (2015). Glycoprotein nonmetastatic melanoma protein B ameliorates skeletal muscle lesions in a SOD1G93A mouse model of amyotrophic lateral sclerosis. J. Neurosci. Res. 93, 1552-1566. doi: 10.1002/jnr.23619
Nalls, M. A., Pankratz, N., Lill, C. M., Do, C. B., Hernandez, D. G., Saad, M., et al. (2014). Large-scale meta-analysis of genome-wide association data identifies six new risk loci for Parkinson's disease. Nat. Genet. 46, 989-993. doi: 10.1038/ng.3043

Nalls, M. A., Plagnol, V., Hernandez, D. G., Sharma, M., Sheerin, U. M., Saad, M., et al. (2011). Imputation of sequence variants for identification of genetic risks for Parkinson's disease: a meta-analysis of genome-wide association studies. Lancet 377, 641-649. doi: 10.1016/S0140-6736(10)62345-8

Neal, M. L., Boyle, A. M., Budge, K. M., Safadi, F. F., and Richardson, J. R. (2018). The glycoprotein GPNMB attenuates astrocyte inflammatory responses through the CD44 receptor. J. Neuroinflamm. 15:73. doi: 10.1186/s12974-018-1100-1

Onaga, M., Ido, A., Hasuike, S., Uto, H., Moriuchi, A., Nagata, K., et al. (2003). Osteoactivin expressed during cirrhosis development in rats fed a cholinedeficient, L-amino acid-defined diet, accelerates motility of hepatoma cells. J. Hepatol. 39, 779-785. doi: 10.1016/S0168-8278(03)00361-1

Pang, S. Y., Ho, P. W., Liu, H. F., Leung, C. T., Li, L., Chang, E. E. S., et al. (2019). The interplay of aging, genetics and environmental factors in the pathogenesis of Parkinson's disease. Transl. Neurodegener. 8:23. doi: 10.1186/s40035-019-0165-9

Pang, S. Y., Teo, K. C., Hsu, J. S., Chang, R. S., Li, M., Sham, P. C., et al. (2017). The role of gene variants in the pathogenesis of neurodegenerative disorders as revealed by next generation sequencing studies: a review. Transl. Neurodegener. 6:27. doi: 10.1186/s40035-017-0098-0

Pihlstrom, L., Axelsson, G., Bjornara, K. A., Dizdar, N., Fardell, C., Forsgren, L., et al. (2013). Supportive evidence for 11 loci from genome-wide association studies in Parkinson's disease. Neurobiol. Aging. 34, 1708.e7-13. doi: 10.1016/j.neurobiolaging.2012.10.019

Postuma, R. B., Berg, D., Stern, M., Poewe, W., Olanow, C. W., Oertel, W., et al. (2015). MDS clinical diagnostic criteria for Parkinson's disease. Mov. Disord. 30, 1591-1601. doi: $10.1002 /$ mds.26424

Prizant, H., Taya, M., Lerman, I., Light, A., Sen, A., Mitra, S., et al. (2016). Estrogen maintains myometrial tumors in a lymphangioleiomyomatosis model. Endocr. Relat. Cancer 23, 265-280. doi: 10.1530/ERC-15-0505

Ripoll, V. M., Irvine, K. M., Ravasi, T., Sweet, M. J., and Hume, D. A. (2007). Gpnmb is induced in macrophages by IFN-gamma and lipopolysaccharide and acts as a feedback regulator of proinflammatory responses. J. Immunol. 178, 6557-6566. doi: 10.4049/jimmunol.178.10.6557

Rose, A. A., Grosset, A. A., Dong, Z., Russo, C., Macdonald, P. A., Bertos, N. R., et al. (2010). Glycoprotein nonmetastatic B is an independent prognostic indicator of recurrence and a novel therapeutic target in breast cancer. Clin. Cancer Res. 16, 2147-2156. doi: 10.1158/1078-0432.CCR-09-1611

Rukavina, K., Leta, V., Sportelli, C., Buhidma, Y., Duty, S., Malcangio, M., et al. (2019). Pain in Parkinson's disease: new concepts in pathogenesis and treatment. Curr. Opin. Neurol. 32, 579-588. doi: 10.1097/WCO.0000000000000711

Saeed, U., Compagnone, J., Aviv, R. I., Strafella, A. P., Black, S. E., Lang, A. E., et al. (2017). Imaging biomarkers in Parkinson's disease and Parkinsonian syndromes: current and emerging concepts. Transl. Neurodegener. 6:8. doi: 10.1186/s40035-017-0076-6

Selvaraj, V. K., and Keshavamurthy, B. (2016). Sleep dysfunction in Parkinson's disease. J. Clin. Diagn. Res. 10, Oc09-12. doi: 10.7860/JCDR/2016/16446.7208

Shen, S. S., Shen, Y., Xiong, K. P., Chen, J., Mao, C. J., Huang, J. Y., et al. (2014). Validation study of REM sleep behavior disorder questionnaire-Hong Kong (RBDQ-HK) in east China. Sleep Med. 15, 952-958. doi: 10.1016/j.sleep.2014.03.020

Shulman, J. M., Yu, L., Buchman, A. S., Evans, D. A., Schneider, J. A., Bennett, D. A., et al. (2014). Association of Parkinson disease risk loci with mild parkinsonian signs in older persons. JAMA Neurol. 71, 429-435. doi: 10.1001/jamaneurol.2013.6222

Soto-Ortolaza, A. I., Heckman, M. G., Labbe, C., Serie, D. J., Puschmann, A., Rayaprolu, S., et al. (2013). GWAS risk factors in Parkinson's disease: LRRK2 coding variation and genetic interaction with PARK16. Am. J. Neurodegener. Dis. 2, 287-299.

Srinivasan, K., Friedman, B. A., Larson, J. L., Lauffer, B. E., Goldstein, L. D., Appling, L. L., et al. (2016). Untangling the brain's neuroinflammatory and neurodegenerative transcriptional responses. Nat. Commun. 7:11295. doi: $10.1038 /$ ncomms 11295 
Tanaka, H., Shimazawa, M., Kimura, M., Takata, M., Tsuruma, K., Yamada, M., et al. (2012). The potential of GPNMB as novel neuroprotective factor in amyotrophic lateral sclerosis. Sci. Rep. 2:573. doi: 10.1038/srep00573

Tsui, K. H., Chang, Y. L., Feng, T. H., Chang, P. L., and Juang, H. H. (2012). Glycoprotein transmembrane nmb: an androgen-downregulated gene attenuates cell invasion and tumorigenesis in prostate carcinoma cells. Prostate 72, 1431-1442. doi: 10.1002/pros.22494

Visser, M., Marinus, J., Stiggelbout, A. M., and Van Hilten, J. J. (2004). Assessment of autonomic dysfunction in Parkinson's disease: the SCOPA-AUT. Mov. Disord. 19, 1306-1312. doi: 10.1002/mds.20153

Weil, R. S., Costantini, A. A., and Schrag, A. E. (2018). Mild cognitive impairment in Parkinson's disease-what is it? Curr. Neurol. Neurosci. Rep. 18:17. doi: 10.1007/s11910-018-0823-9

Williams-Gray, C. H., Mason, S. L., Evans, J. R., Foltynie, T., Brayne, C., Robbins, T. W., et al. (2013). The CamPaIGN study of Parkinson's disease: 10-year outlook in an incident population-based cohort. J. Neurol. Neurosurg. Psychiatry. 84, 1258-1264. doi: 10.1136/jnnp-2013-305277

Xu, Y., Chen, Y., Ou, R., Wei, Q. Q., Cao, B., Chen, K., et al. (2016). No association of GPNMB rs156429 polymorphism with Parkinson's disease, amyotrophic lateral sclerosis and multiple system atrophy in Chinese population. Neurosci. Lett. 622, 113-117. doi: 10.1016/j.neulet.2016.04.060
Zambito Marsala, S., Tinazzi, M., Vitaliani, R., Recchia, S., Fabris, F., Marchini, C., et al. (2011). Spontaneous pain, pain threshold, and pain tolerance in Parkinson's disease. J. Neurol. 258, 627-633. doi: 10.1007/s00415-010-5812-0

Zhou, L. T., Liu, F. Y., Li, Y., Peng, Y. M., Liu, Y. H., and Li, J. (2012). Gpnmb/osteoactivin, an attractive target in cancer immunotherapy. Neoplasma 59, 1-5. doi: 10.4149/neo_2012_001

Zhuo, H., and Zhou, L. (2016). Gpnmb/osteoactivin: an indicator and therapeutic target in tumor and nontumorous lesions. Die. Pharmazie 71, 555-561. doi: $10.1691 /$ ph.2016.6683

Conflict of Interest: The authors declare that the research was conducted in the absence of any commercial or financial relationships that could be construed as a potential conflict of interest.

Copyright (c) $2020 \mathrm{Liu}, \mathrm{Li}, \mathrm{He}$, He, Zhang, Shen, Zhang, Chen, Cui and Tan. This is an open-access article distributed under the terms of the Creative Commons Attribution License (CC BY). The use, distribution or reproduction in other forums is permitted, provided the original author(s) and the copyright owner(s) are credited and that the original publication in this journal is cited, in accordance with accepted academic practice. No use, distribution or reproduction is permitted which does not comply with these terms. 